\title{
Evaluación del ambiente educacional a través del cuestionario validado DREEM entre dos Universidades españolas
}

\section{Evaluation of the educational environment through the DREEM validaded questionnaire between two Spanish Universities}

\author{
Mercedes Ruidiaz Peña ${ }^{1}$, Ana $\mathrm{M}^{\mathrm{a}}$ Gáscon Catalán ${ }^{1}$, Eva $\mathrm{M}^{\mathrm{a}}$ Gómez Trullén ${ }^{1}$,Emilio Fco. Ignacio Garcia ${ }^{2}$, Ana \\ Martinez Martinez ${ }^{1}$, Clara Alcaine Gonzalez ${ }^{1}$, Delia Gonzalez de la Cuesta ${ }^{1}$ \\ mruidiaz@unizar.es, agascon@unizar.es,egomez@unizar.es,Emilio.ignacio@uca.es, amarmar@unizar.es, necaila@unizar.es , \\ delia@unizar.es \\ ${ }^{1}$ Fisiatría y Enfermería \\ Universidad de Zaragoza \\ Zaragoza, Cádiz \\ ${ }^{2}$ Fisiterapía y Enfermería \\ Universidad de Cádiz \\ Cádiz, España
}

\begin{abstract}
Resumen- Estudio centrado en comparar la percepción del Ambiente educacional de estudiantes de $4^{\circ}$ curso del grado de Enfermería en dos Universidades públicas, de Zaragoza y Cádiz. Estudio descriptivo transversal. La percepción del Ambiente educacional se midió a través del cuestionario Dundee Ready Educación Environment Measure validado para Ciencias de la salud. El número total de encuestas obtenidas fue de 170. Para ver diferencias en la percepción del aprendizaje y la adquisición de competencias profesionales se empleó la t-student y se analizó si existía correlación mediante el coeficiente de correlación de Pearson. Los estadísticos se realizaron con el SPSS v22. Los resultados descriptivos muestran, edad mayoritariamente entre $18-21$ años, un $86 \%$ de mujeres y un $62,5 \%$ eligen como $1^{\text {a }}$ opción Enfermería. El 78,8\% de estudiantes percibe el "Ambiente como adecuado" sin observarse diferencias entre las dos Universidades. Respecto a las competencias profesionales la valoración media es mayor en la Universidad de Zaragoza que en la de Cádiz $(\mathrm{p}<0,001)$ también existe una correlación positiva en la Universidad de Zaragoza entre competencias profesionales con la percepción global del Ambiente educacional de los estudiantes $(p<0,05)$.El proyecto es sostenible y transferible, debemos profundizar en implantar mejoras para la adquisición de competencias profesionales en el grado de Enfermería.
\end{abstract}

Palabras clave: Ambiente educacional, Precepción de los estudiantes, Estudiantes de ciencias de la salud, cuestionario DREEM

Abstract- Study focused on comparing the perception of the educational environment of students in the 4th year of the Nursing degree in two public universities, Zaragoza and Cádiz. Descriptive cross-sectional study. The perception of the educational environment was measured through the Dundee Ready Education Environment Measure validated questionnaire for health sciences. The total number of surveys obtained was 170 . To see differences in the perception of learning and the acquisition of professional skills, the t-student was used and it was analyzed whether there was a correlation using the Pearson correlation coefficient. The statistics were made with SPSS v22. The descriptive results show, mostly between $18-21$ years old, $86 \%$ of women and $62.5 \%$ choose as 1 st option Nursing. $78.8 \%$ of students perceive the "Environment as adequate" without observing a difference between the two Universities. Regarding professional competences, the average assessment is higher in the University of Zaragoza than in Cádiz $(p<0.001)$ there is also a positive correlation in the University of Zaragoza between professional competences with the overall perception of the students' educational environment $(\mathrm{p}$ $<0.05$ ). The project is sustainable and transferable, we must deepen in implementing improvements for the acquisition of professional competences in the Nursing degree.

Keywords: Educational environment, Perception of the students, Students of health sciences, DREEM questionnaire

\section{INTRODUCCIÓN}

El ambiente educacional (AE) es un aspecto importante a tener en cuenta en la educación de Ciencias de la salud. Su relevancia ha sido reconocida y aceptada por su influencia sobre la satisfacción y éxito de los estudiantes, el logro académico se asocia positivamente con la percepción de su entorno de aprendizaje. (Genn, 2001; Díaz, et al., 2002; Herrera, et al., 2012; Al-Qahtani, 2015).

Se han descritos dos grupos de factores que influencian el AE: cursos-currículo y docentes. El primero está compuesto por el estilo curricular, calidad de enseñanza, señalización y claridad de los procesos, resultados, evaluaciones y mecanismos de apoyo. El segundo se relaciona con estilostécnicas de enseñanza, ambiente físico y modelos a seguir. Estos factores llevan a la motivación, relevancia percibida y sentido del deber por el estudiante, lo que finalmente influye en los resultados de su aprendizaje. (Díaz, et al., 2002).

Un AE desfavorable puede conducir a una percepción de insatisfacción y frustración que repercutiría en el rendimiento académico del estudiante. Por el contrario, los estudiantes que perciben más favorablemente el ambiente educacional logran mayores éxitos académicos, lo cual les genera mayor satisfacción y mejor rendimiento académico. (Hutchinson, 2003).Esto tiene importancia puesto que puede generar menos estrés, angustia, y abandono de la carrera por parte de los estudiantes (Soemantri, Herrera y Riquelme, 2010). 
Para medir el AE existen distintos instrumentos en el área de Ciencias de salud, tanto para el aula como para ambientes clínicos. (Roff, 2005).Entre los instrumentos disponibles para medir el ambiente educacional en pregrado, el cuestionario DREEM (Dundee Ready Education Environment Measure) ha sido reconocido como uno de los instrumentos más fiables (Soemantri, et al., 2010) validado en 1997 para Ciencias de la salud. (Roff, et al., 1997).

Este cuestionario se recomienda para obtener el perfil de los estudiantes de Medicina y Enfermería, identificando sus fortalezas y debilidades, pudiendo además realizar un análisis comparativo de la percepción de estos, en una misma institución o entre instituciones, evaluar la correlación entre percepción del Ambiente educacional y logros académicos de los alumnos . (Roff, 2005).

Debido a la heterogeneidad de titulaciones en los grados de Enfermería en el territorio español, hemos analizado y comparado el Ambiente educacional, en el grado de Enfermería entre dos Universidades públicas españolas.

Comprendiendo las diferencias geográficas, socio-culturales y económicas entre las dos Universidades hemos analizado la percepción enseña-aprendizaje que tienen los estudiantes tras la implantación del plan Bolonia en el modelo curricular. Deseamos detectar el "problema raíz" para intervenir a través de la innovación docente, por medio de participaciones activas, implicando al alumno y profesor, para mejorar las competencias de la titulación del grado de Enfermería

\section{CONTEXto}

La formación en Enfermería, se ha ido adaptando a los cambios acaecidos en el contexto de las Ciencias de la Salud.

La integración en 1977 de los estudios de Enfermería en la Universidad. (Real Decreto 2128/1977) supuso un punto de inflexión en la transición de la disciplina enfermera desde una etapa técnica a una profesional. A lo largo de la década de los 80 la Enfermería empieza a consolidarse como disciplina y las distintas corrientes profesionales trabajan en el desarrollo de un cuerpo de conocimientos propio, que proporcione un nuevo marco de definición de la responsabilidad de las enfermeras/os en la atención a la salud de la población.

En estos años, la formación de Enfermería, que hasta ese momento había sido eminentemente práctica, da un giro sustancial en su diseño curricular planteando un peso equivalente en la distribución de teoría y práctica clínica. El inicio de la década de los 90 marca la segunda etapa de la formación de Enfermería, ya que como resultado del desarrollo de la Ley de Reforma Universitaria (LRU), se publican las nuevas directrices para la elaboración de los planes de estudios de los Diplomados en Enfermería (Real Decreto 1466/1990).

El proceso de cambio que sufre la Universidad queda plasmado, entre otros aspectos, por el mayor protagonismo del estudiante en el proceso de enseñanza-aprendizaje. Es destacable también en esta etapa el cambio producido en la metodología educativa, que asigna un papel más activo al estudiante y dirige sus esfuerzos al desarrollo de un pensamiento más reflexivo.

De esta forma, los estudios de la diplomatura en Enfermería empiezan a estar en consonancia con la normativa europea (Directiva del Consejo 89/595/CEE de 10 de octubre de 1989), que propugna la libre circulación y el intercambio de profesores y estudiantes en el marco comunitario y se da un especial impulso a los programas Erasmus.

En España los estudios de Enfermería pasaron a ser estudios de Grado en el año 2010.

\section{A. Objetivos}

1. Valorar la percepción de los estudiantes del ambiente educacional de $4^{\circ}$ curso de grado de enfermería en dos universidades públicas

2. Comparar la percepción de los estudiantes del ambiente educacional de las dos universidades del grado de enfermería al finalizar los estudios universitarios

3. Obtener el perfil del ambiente educacional en el grado de enfermería, evaluando fortalezas y debilidades tal y como son percibidas por los estudiantes para la posible intervención de los profesores y sistema educativo

\section{DESCRIPCIÓN}

\section{A. Tipo de estudio y población:}

Estudio descriptivo transversal centrado en comparar la percepción de los estudiantes de $4^{\circ}$ del grado de Enfermería acerca del ambiente educacional en dos Universidades públicas. Se utilizó un muestreo no probabilístico por conveniencia para el reclutamiento de alumnos, con un total de 170 participantes, siendo 133 de la Universidad de Zaragoza y 37 de la Universidad de Cádiz. Los cuestionarios los cumplimentaron las últimas semanas del primer cuatrimestre. A todos los alumnos se les informó de los objetivos de la investigación, se solicitó su participación y colaboración. Se explicó que los cuestionarios eran anónimos y voluntarios y que se requería aproximadamente 10 minutos para su realización.

\section{B. Herramienta utilizada cuestionario DREEM:}

Esta escala esta validada para los estudios de Ciencias de la salud por Roff et al. (1997) y traducida al español. Consta de 50 ítems tipo Likert en una escala de 5 puntos $(0$, muy en desacuerdo; 1, parcialmente en desacuerdo; 2, inseguro/dudoso; 3, parcialmente de acuerdo, y 4, totalmente de acuerdo). Los 50 ítems cubren aspectos relevantes para el ambiente educacional y se dividen en cinco Dominios:

Dominio 1.Percepción de la enseñanza, 12 items (máx48 puntos)

- Pregunta.1.Se me estimula a participar en clases

- Pregunta.7.La enseñanza es frecuentemente estimulante

- Pregunta.13.La enseñanza está centrada en el estudiante

- Pregunta.16.La enseñanza me ayuda a desarrollar mi competencia

- Pregunta.20.La enseñanza está bien enfocada 
- Pregunta.22.La enseñanza en la escuela está suficientemente preocupada de desarrollar mi confianza

- Pregunta24.El tiempo destinado a la enseñanza es bien utilizado

- Pregunta.25.La enseñanza en la escuela pone demasiado énfasis en el aprendizaje de detalles

- Pregunta.38.Tengo claros los objetivos de aprendizaje de mis cursos

- Pregunta.44.La manera de enseñar me estimula a aprender por mí mismo en forma activa

- Pregunta.47.En la escuela, se enfatiza el aprendizaje a largo plazo por sobre el inmediato

- Pregunta.48.La enseñanza de la escuela está demasiado centrada en los docentes

Dominio 2.Percepción de los docentes, 11 items (máx44 punto).

- Pregunta.2.Los docentes conocen las materias que dictan

- Pregunta.6.Los docentes tienen paciencia con los pacientes

- Pregunta.8.Los docentes ridiculizan a los estudiantes

- Pregunta.9.Los docentes son autoritarios

- Pregunta.18.Los docentes tienen buenas destrezas comunicacionales con los pacientes

- Pregunta.29.Los docentes son buenos dando feedback (retroalimentación) a los estudiantes

- Pregunta.32.En la escuela, los docentes nos hacen críticas constructivas

- Pregunta.37.Los docentes dan ejemplos claros

- Pregunta.39.Los docentes se molestan y alteran en clases

- Pregunta.40. Los docentes están bien preparados para sus clases

- Pregunta.50.Los estudiantes causamos irritación a los docentes

Dominio 3.Autopercepción académica, 8 items,(máx32 puntos)

- Pregunta.5.Los métodos de estudio que tenía antes todavía me sirven

- Pregunta.10.Tengo la confianza de que voy a pasar este año

- Pregunta.21.Siento que me están preparando bien para mi profesión

- Pregunta.26.Lo aprendido el año pasado fue una buena base para el trabajo de este año

- Pregunta.27.Soy capaz de memorizar todo lo que me es necesario
- Pregunta.31.He aprendido mucho sobre la empatía en mi profesión

- Pregunta.41.La escuela de Enfermería me ayuda a desarrollar mis destrezas para resolver problemas

- Pregunta.45.Mucho de lo que tengo que aprender me parece relevante para mi carrera

Dominio 4.Percepción del aprendizaje, 12 items (máx48 puntos)

- Pregunta.11.El ambiente es relajado durante las visitas docentes de los servicios hospitalarios

- Pregunta.12.Los horarios de la escuela están bien programados

- Pregunta.17.En la escuela, la copia en los exámenes constituye un problema

- Pregunta.23.El ambiente es relajado durante las clases teóricas en el auditorio

- Pregunta.30.Tengo oportunidades para desarrollar mis habilidades interpersonales

- Pregunta.33.Me siento cómodo, socialmente, en clases

- Pregunta.34.El ambiente en los seminarios, clases y prácticas tutoriales es relajado

- Pregunta.35.Mi experiencia en la escuela ha sido desalentadora

- Pregunta.36.Soy capaz de concentrarme bien

- Pregunta.42.El disfrute de mis estudios en la escuela pesa más que la tensión que estos me generan

- Pregunta.43.El ambiente de la escuela me motiva a aprender

- Pregunta.49.Siento que puedo hacer todas las preguntas que quiero

Dominio 5.Autopercepción social, con 7 items,(máx28 puntos)

- Pregunta.3.Hay un buen sistema de apoyo para los estudiantes que sufren de estrés

- Pregunta.4.Estoy demasiado cansado para disfrutar los cursos que estoy tomando

- Pregunta.14.Rara vez me aburro en los cursos que estoy tomando

- Pregunta.15.Tengo buenos amigos en la escuela

- Pregunta.19.Mi vida social es buena

- Pregunta.28.Rara vez me siento solo

- Pregunta.46.Los ambientes físicos de la escuela son agradables

Al sumar las puntuaciones de los 50 ítems se obtiene un máximo total de 200 puntos. La puntuación final puede interpretarse de la siguiente manera: 0-50 puntos, ambiente educacional considerado como "muy pobre". 51-100 puntos, ambiente educacional con "muchos problemas". 101-150 puntos, ambiente educacional con "más aspectos positivos que 
negativos" (adecuado). 151-200 puntos, ambiente educacional "excelente".

La interpretación de las puntuaciones totales y de cada dominio o subescala se realizó según lo sugerido por McAleer y $\operatorname{Roff}(2001)$.

\section{Herramienta utilizada cuestionario preguntas propias}

Al cuestionario DREEM se adjuntó 3 preguntas respecto a la edad, sexo y $1^{\mathrm{a}}$ opción para la elección de carrera y 5 preguntas sobre competencias profesionales, en una escala analógica de 0 a 10 puntos.

Pregunta.1.Como te sientes de preparado para trabajar de enfermera/ro

Pregunta.2.Como te sientes de preparado en habilidades técnicas ejemplo: extracción de sangre, curas...

Pregunta.3.Como te sientes de preparado a nivel de conocimientos teóricos

Pregunta.4.Como te sientes de preparado para desarrollar el proceso enfermero ejemplo: valorar, elaborar plan de cuidados

Pregunta.5.Como te sientes de preparado para trabajar en equipo

\section{Tratamiento estadístico:}

Para el análisis de los resultados se utilizó el paquete estadístico SPSS versión 22. En primer lugar se hizo un análisis descriptivo de la muestra en porcentajes y frecuencias para las variables cualitativas y en medias y desviaciones estándar para las cuantitativas. Para ver si había diferencias en la percepción del aprendizaje o de la adquisición de competencias profesionales se realizó el estadístico test de Student. Además se estudió si existía una correlación lineal entre la percepción del ambiente educacional y la adquisición de competencias con el coeficiente de correlación de Pearson. Se consideró que existía significancia estadística si $(\mathrm{p}<0,05)$

\section{Resultados}

\section{A. Descripción de la población de estudio:}

La distribución por edades de la población total a estudio no ha mostrado diferencia entre las dos Universidades, estando comprendida principalmente por estudiantes de entre 18-21 años, en un $69,4 \%$.Respecto al sexo, la mayor parte son mujeres, en un $86 \%$ del total, no habiendo tampoco diferencias entre las dos Universidades. Un $62,5 \%$ eligieron como $1^{\text {a }}$ opción los estudios de Enfermería al solicitar la admisión en la Universidad, sin observarse destacadas diferencias entre las dos Universidades, pero el 37,5\% de los jóvenes menores de 21 años no eligieron Enfermería en $1^{\mathrm{a}}$ opción, siendo ligeramente superior en la Universidad Zaragoza frente a la de Cádiz.

No es desdeñable y queremos resaltar el porcentaje de jóvenes de entre 18-21 años, que desean hacer Medicina en lugar de Enfermería. Al cuestionario DREEM adjuntamos tres preguntas más: edad, sexo y $1^{\mathrm{a}}$ opción. Respecto a esta última pregunta, los profesores llevábamos detectando en nuestra trayectoria como docentes, el hecho de que bastantes alumnos deseaban hacer Medicina en lugar de Enfermería. A nuestro entender ciertos alumnos, seguramente, con alta nota de Selectividad prefieren hacer Medicina en $1^{\text {a }}$ opción, contribuyendo, en ocasiones a cierto malestar entre los alumnos, lo que podría suponer peor Ambiente educacional. Es de lamentar no poder comparar este problema con otras Universidades ya que el cuestionario DREEM no se ha evaluado en ninguna otra Universidad española en el grado de Enfermería .

La valoración del Ambiente educacional se agrupo en cuatro categorías: Ambiente pobre. Ambiente con bastantes problemas. Ambiente adecuado y Ambiente excelente. En ninguna de las dos Universidades se ha detectado Ambiente pobre. La gran mayoría de los estudiantes, un 78,8\% valoraron el Ambiente como adecuado, no habiendo diferencia entre las dos Universidades. Sin embargo Ambiente con bastantes problemas lo detecta un porcentaje mayor los estudiantes de la Universidad de Cádiz frente a la Universidad de Zaragoza, $21,6 \%$ vs $12 \%$

B. Medias del Ambiente educacional Global y por Dominios de $4^{\circ}$ curso del grado de Enfermería en la población a estudio de las dos Universidades:

El Ambiente es "adecuado" para la población general. Por Universidades es más alto en todos los dominios en la Universidad de Zaragoza, pero no existen diferencias significativas entre ellas. La media obtenida en los distintos dominios indica una percepción positiva (Tabla 1).

Tabla 1:

Ambiente educacional global y por dominios

\begin{tabular}{ccccl}
\hline & Total & U. Zaragoza & U. Cádiz & p \\
& & & & \\
\hline D1 & $27,42 \pm 5,97$ & $27,56 \pm 5,73$ & $26,92 \pm 6,8$ & $\mathrm{~ns}$ \\
D2 & $27,52 \pm 5,89$ & $27,93 \pm 5,65$ & $26,03 \pm 6,54$ & $\mathrm{~ns}$ \\
D3 & $22,94 \pm 4,18$ & $23,2 \pm 4,13$ & $22 \pm 4,3$ & $\mathrm{~ns}$ \\
D4 & $29,1941 \pm 3,83$ & $29,01 \pm 5,37$ & $28,43 \pm 6,04$ & $\mathrm{~ns}$ \\
D5 & $16,38 \pm 4,61$ & $16,31 \pm 3,61$ & $16,38 \pm 4,61$ & $\mathrm{~ns}$ \\
G & $123,40 \pm 21,17$ & $124,41 \pm 19,98$ & $119,76 \pm 24,97$ & $\mathrm{~ns}$ \\
\hline
\end{tabular}

Nota: D1: Percepción de la enseñanza; D2: Percepción de los docentes; D3: Autopercepción académica; D4: Percepción del aprendizaje; D5: Autopercepción social; G: global; ns: no significativo

De acuerdo con nuestro planteamiento nuestros resultados respecto al Ambiente educacional global tanto en Zaragoza como en Cádiz, son similares a los estudios de Cáneo, Brizuela, Muñoz, Pérez y Solsona (2016) que obtienen en la Universidad Diego Portales de Santiago de Chile, en $4^{\circ}$ curso de Enfermería, un valor medio de 125,8 $\pm 20,9$

También es similar al grado de Kinesiologia en la Universidad Pontificia Católica de Chile, donde el promedio global del cuestionario DREEM de Fuenzalida et al. (2018) fue de $135,74 \pm 119,15$

Es decir, nuestros resultados son ligeramente inferiores pero se encuentran dentro del rango de 101-150 puntos, correspondiente a una percepción global del Ambiente educacional adecuado. Siendo, no obstante Cádiz la de menor valor alcanzado.

Respecto a los Dominios, nuestros resultados tanto de Zaragoza como de Cádiz, son también similares a los obtenidos en la Universidad Diego Portales, para los dominios 1, 2, 3 y 5. En cambio, en el dominio 3, en la Universidad de 
Zaragoza el valor medio fue de $23,2 \pm 4,13$ y en Cádiz de $22 \pm 4,3$ obteniendo en ambas Universidades de nuestro país mayor puntaje que nuestros colegas de la Universidad Diego Portales 21 44,13 (Cáneo, et al., 2016).

\section{Valoración de "preguntas propias" fuera del} cuestionario DREEM realizadas al alumnado:

La valoración subjetiva de los estudiantes de $4^{\circ}$ curso del grado, respecto a cómo se consideraban de preparados, para trabajar como enfermeras/os, se realizó con 5 preguntas adicionales, elaboradas por el equipo de investigación considerándolas relevantes para evaluar la formación académica recibida. La valoración global es media/alta con una puntuación de 7 en casi todos los apartados. En estas valoraciones la media es más alta en la Universidad de Zaragoza con diferencias significativas entre las dos Universidades, por lo tanto subjetivamente los alumnos se sienten más preparados en la Universidad de Zaragoza que en la de Cádiz (Tabla 2)

Tabla 2:

Autopercepción de competencias profesionales

\begin{tabular}{llccc}
\hline & Total & $\begin{array}{c}\text { U. } \\
\text { Zaragoza }\end{array}$ & U. Cádiz & p \\
\hline P1 & $7,06 \pm 1,65$ & $7,36 \pm 1,49$ & $5,94 \pm 1,72$ & 0,0001 \\
P2 & $7,83 \pm 1,66$ & $8,3 \pm 1,15$ & $6,08 \pm 2,08$ & 0,0001 \\
P3 & $6,9 \pm 1,57$ & $7,17 \pm 1,43$ & $5,94 \pm 1,72$ & 0,0001 \\
P4 & $6,64 \pm 1,78$ & $6,87 \pm 1,6$ & $5,78 \pm 2,15$ & 0,001 \\
P5 & $7,37 \pm 1,58$ & $7,68 \pm 1,32$ & $6,24 \pm 1,91$ & 0,0001
\end{tabular}

Nota: P1: Trabajo enfermería; P2: Habilidades técnicas; P3: Conocimientos teóricos; P4: Proceso enfermero; P5: Trabajo equipo.

Aunque la diferencia sea estadísticamente significativa entre las dos Universidades, es un resultado que deberemos de tomarlo con cautela ya que la muestra de alumnos obtenida en Cádiz, es menor que en Zaragoza. Las 5 preguntas incorporadas por los docentes se debieron de nuevo a motivos observacionales, para valorar como se sienten preparados tras el paso por la Facultad antes de incorporarse al mundo laboral, ya que los alumnos de $4^{\circ}$ curso nos suelen transmitir en diversas ocasiones esta preocupación. Este test de competencias profesionales de elaboración propia se halla en fase de desarrollo para su validación y no hay posible comparación con otros autores.

D. Correlación de "preguntas propias" fuera del cuestionario DREEM con el Ambiente educacional global de los estudiantes:

Las preguntas: Preparado para trabajar como enfermero, Preparado a nivel de conocimientos teóricos, Preparado para realizar un proceso enfermero y Preparado para trabajar en equipo, correlacionan positivamente en la Universidad de Zaragoza con el Ambiente educacional global, $(\mathrm{p}<0,001, \mathrm{p}$ $<0,0001, \mathrm{p}<0,001$ y $\mathrm{p}<0,0001)$ y no correlaciona la pregunta, Preparado con habilidades técnicas. Sin embargo en la Universidad Cádiz solo la pregunta, Preparado a nivel de conocimientos teóricos, correlaciona levemente $\mathrm{p}<0,037$ con el Ambiente educacional global. Lo cual podría explicar el porcentaje más elevado de detección de: Ambiente con bastantes problemas en la Universidad de Cádiz 21,6\% frente a la Universidad de Zaragoza de 12\%

No hemos encontrado bibliografía que nos aporte información respecto a la correlación entre preguntas de competencias profesionales con el Ambiente educacional global y solo Cáneo (2016) muestra una correlación positiva $\mathrm{p}<0,0001$ de la percepción individual de los estudiantes entre los distintos dominios, sugiriendo que existen alumnos con una percepción más positiva en general y otros más negativa, según dicha autora la percepción que tenga el estudiante de la enseñanza influye significativamente en todos los dominios.(Cáneo, et al.,2016)

Queremos destacar la correlación evidenciada entre el Ambiente educacional y la adquisición de competencias profesionales, demostrando la importancia de tener un buen Ambiente educacional en todas sus dimensiones, para obtener éxitos en la formación de estos profesionales, sintiéndose mejor preparados, los que durante su formación han percibido un mejor clima en el proceso de enseñanza aprendizaje. Es por tanto un aspecto a tener en cuenta, ya que es además fácilmente evaluable y permite detectar debilidades, fortalezas y proponer mejoras que repercutirán en la formación final de estos profesionales sanitarios. En general los alumnos se sienten competentes para realizar el trabajo de enfermería, valorando tanto su preparación a nivel de conocimientos teóricos, habilidades técnicas, para realizar un proceso enfermero y para trabajar en equipo. Sin embargo, en estas preguntas referidas a cómo se sienten preparados al finalizar la carrera para incorporarse al mundo laboral, hemos detectado peor valoración en la Universidad de Cádiz que en la de Zaragoza, pero puede atribuirse esta diferencia al pequeño número muestral de la Universidad de Cádiz. Sería deseable aumentar el número de participantes de esta Universidad fortaleciendo la metodología de reclutamiento de estudiantes por parte de los profesores para obtener más respuestas y confirmar si realmente ha habido sesgo o no.

\section{CONCLUSIONES}

Los resultados ponen de manifiesto que los estudiantes al finalizar los estudios del grado en Enfermería perciben que el Ambiente educacional en el que han realizado sus estudios, ha sido adecuado tanto a nivel global como en lo relativo a la enseñanza, a los docentes, a la autopercepción académica, a la percepción del aprendizaje y a la autopercepción social. El perfil del alumnado es muy similar en las dos Universidades, correspondiendo en su mayoría a jóvenes de entre 18-21 años, mujeres, y que eligieron Enfermería en $1^{\circ}$ opción al iniciar sus estudios universitarios

Esta investigación es un proyecto sostenible debido $\mathrm{s}$ experiencias previas que nos han permitido evaluar el Ambiente educacional a lo largo de los estudios de Enfermería en la Universidad pública de Zaragoza. Es eficiente por no requerir elevado coste económico y el cuestionario DREEM, es una herramienta fiable y resulta útil para conocer cómo perciben el Ambiente educacional los estudiantes de Enfermería y comparar con distintas Universidades españolas. Nos permite detectar áreas de mejora del proceso enseñanzaaprendizaje y esto motiva próximas investigaciones para 
explorar las competencias profesionales específicas del grado de Enfermería

\section{AgRADECIMIENTOS}

Al Vicerrectorado de política académica de la Universidad pública de Zaragoza por la subvención del proyecto de innovación docente PIIDUZ 18 211titulado: Evaluación del Ambiente educacional y aprendizaje teórico-práctico en el grado de Enfermería de Zaragoza y Cádiz.

\section{REFERENCIAS}

Al-Qahtani, M.F. (2015). Associations between approaches to study, the learning environment, and academic achievement. Journal of Taibah University Medical Sciences, 10(1), 56-65.

Cáneo, M., Brizuela, M.J., Muñoz, P., Pérez. G., Solsona D. (2016). Percepción del ambiente educacional preclínico en estudiantes de $1^{\circ}$ a $4^{\circ}$ año de enfermería de la Universidad Diego Portales, Santiago (Chile). Revista Iberoamericana de Educación e Investigación en Enfermería, 6(2), 37-45.

Díaz, M., Peio, U., Arias, J., Escudero, T., Rodriguez. S., Vidal, J. (2002). Evaluación del rendimiento en la enseñanza superior. Comparación de resultados entre alumnos procedentes de la Logse y del Cou. Revista de Investigación Educativa, 20, 357-83.

Directiva del Consejo 89/595/CEE de 10 de octubre, por la que se modifica la Directiva $77 / 452 / \mathrm{CEE}$ sobre el reconocimiento recíproco de los diplomas, certificados y otros títulos de enfermero responsable de cuidados generales, que contiene además medidas destinadas a facilitar el ejercicio efectivo del derecho de establecimiento y libre prestación de servicios, así como la Directiva 77/453/CEE sobre coordinación de las disposiciones legales, reglamentarias y administrativas relativas a las actividades de los enfermeros responsables de cuidados generales. Diario oficial de las Comunidades Europeas $n^{\circ} L 341 / 30$ de 23/11/1989 p. 0030 -0032.

Fuenzalida, B., Pizarro, M., Fuentes, J., San Martín, C., Rojas, V., López-Fuenzalida, A., Padilla, O., Riquelme, A. (2018). Percepción del ambiente educacional en estudiantes de pregrado de la carrera de Kinesiología: metodología mixta. Educación Médica. https://doi.org/10.1016/j.edumed.2018.05.010

Genn, J.M. (2001). AMEE Medical Education Guide No. 23 (Part 1): curriculum, environment, climate, quality and change in medical education: a unifying perspective. Medical Teacher, 23(4), 337-44.

Herrera, C.A., Olivos, T., Roman, J.A., Larraín, A., Pizarro, M., Solís, N. et al. (2012). Evaluación del ambiente educacional en programas de especialización médica. Revista Médica de Chile, 140, 1554-61.

Hutchinson, L. (2003). Abc of learning and teaching: educational environment. BMJ. 326 (7393), 810-2.

McAleer, S., y Roff, S. (2001). A practical guide to using the Dundee Ready Education Environment Measure (DREEM). In Genn JM, ed. Curriculum, environment, climate, quality and change in medical education: a unifying perspective. AMEE Education Guide No 23. Dundee: Association for Medical Education in Europe. p. 29-33

Real Decreto 2128/1977, de 23 de julio, sobre integración en la Universidad de las Escuelas de Ayudantes Técnicos Sanitarios como Escuelas Universitarias de Enfermería. $B$ BOE núm. 200, (1977).

Real Decreto 1466/1990, de 26 de octubre, por el que se establece el título universitario oficial de Diplomado en Enfermería y las directrices generales propias de los planes de estudios conducentes a la obtención de aquél. $B$ BOE núm. 278, (1990).

Roff, S., McAleer, S., Harden, R.M., Al-Qahtani, M., Ahmed, A.U., Deza, H. et al. (1997). Development and validation of the Dundee Ready Education Environment Measure (DREEM). Medical Teacher, 19, 295-9

Roff, S. (2005). The Dundee Ready Educational Environment Measure (DREEM): a generic instrument for measuring student's perceptions of undergraduate health professions curricula. Med Teach. 27(4):322-5.

Soemantri, D., Herrera, C., Riquelme, A., (2010). Measuring the educational environment in health professions studies: a systematic review. Medical Teacher, 32(12), 947 